\title{
Author's reply to comment on "The effect of photodynamic therapy on tumor angiogenesis. Cellular and Molecular Life Sciences, 66, 2275-2283"
}

\author{
Ramaswamy Bhuvaneswari • Yik Yuen Gan • \\ Khee Chee Soo $\cdot$ Malini Olivo
}

Received: 26 January 2010/ Accepted: 26 January 2010/Published online: 10 March 2010

(C) Springer Basel AG 2010

The comments by Nowak-Slieinska et al. are an extension of our review article. We agree with the authors that vascular normalization is an important event that decides the outcome of anti-angiogenesis therapies. As reported by Jain RK et al. [1], antiangiogenic agents can normalize the abnormal structure and function of tumor vasculature to make it more efficient for oxygen and drug delivery. Therefore, induction of vascular normalization caused by anti-angiogenic agents provides a novel means of effective delivery of chemotherapeutic drugs. Our report was based on the effective use of anti-angiogenesis therapy to improve therapeutic efficacy of PDT. The authors agree that the use of anti-angiogenesis agents before PDT to induce vascular normalization and increase the homogenous effect of oxygen-dependent PDT is an interesting hypothesis that is worth pursuing.

\section{Reference}

1. Jain RK (2005) Normalization of tumor vasculature: an emerging concept in antiangiogenic therapy. Science 307(5706):58-62 Review

R. Bhuvaneswari $\cdot$ K. C. Soo $\cdot$ M. Olivo $(\bowtie)$

Division of Medical Sciences,

National Cancer Centre Singapore,

Lab 5 Level 511 Hospital Drive,

Singapore 169610, Singapore

e-mail:dmsmcd@nccs.com.sg

Y. Y. Gan

National Institute of Education,

Natural Sciences and Science Education,

Singapore, Singapore

M. Olivo

Biomedical Sciences Institutes,

Singapore Bioimaging Consortium,

Singapore, Singapore

M. Olivo

Department of Pharmacy,

National University of Singapore,

Singapore, Singapore 